UDK 630*453 Lymantria dispar (L.):630*411.1(497.11-191.2)=111

Original scientific paper

\title{
GYPSY MOTH, Lymantria dispar (L.), AND ITS NATURAL ENEMIES IN THE FORESTS OF CENTRAL SERBIA
}

\author{
Mara TABAKOVIĆ-TOŠIĆ ${ }^{1}$
}

\begin{abstract}
In central Serbia, a total of 88 species which are natural enemies of the gypsy moth, i.e. 23 predators, 49 parasitoid insects and 10 saprophagous insects, and 6 pathogens, has been reported. The most abundant of them are the insects which attack the gypsy moth in the larval instar (41 species). Regarding the number of the species, the representatives of the Hymenoptera (14 species from Ichneumonidae family and 11 species from Braconidae family) and Diptera orders $(12$ species from Tachinidae family and 8 species from Sarcophagidae family) are most frequent. Regarding the predators of the gypsy moth, Carabidae family, from Coleoptera order, is most frequent. In addition, at some sites Lymantria dispar nucleopolyhedrosis virus and Entomophaga maimaiga had the dominant role in the reduction of the gypsy moth density.
\end{abstract}

Key words: the gypsy moth, predators, parasitoids, pathogens

\section{GUBAR, Lymantria dispar (L.), I NJEGOVI PRIRODNI NEPRIJATELJI U ŠUMAMA CENTRALNE SRBIJE}

Izvod: U centralnoj Srbiji do danas je utvrđeno ukupno 88 vrste prirodnih neprijatelja gubara, $i$ to 23 predatora, 49 parazitoida, 10 vrsta koje se ponašaju kao saprofagi i parazitoidi, te 6 patogena. U navedenom broju najzastupljeniji su insekti koji parazitiraju larveni stadijum gubara (41 vrsta). Po broju zastupljenih vrsta, najviše je pripadnika redova Hymenoptera (12 vrsta iz familije Ichneumonidae i 11 iz familije Braconidae) i Diptera (12 vrsta iz familije Tachinidae i 8 iz Familije Sarcophagidae). Od predatora gubara, najzastupljenija ja familija Carabidae iz reda Coleoptera. Takođe, $u$ pojedinim područjima Lymantria dispar nucleopolyhedrosis virus i entomopatogena gljiva

${ }^{1} \mathrm{Ph} . \mathrm{D}$ Mara Tabaković-Tošić, Principal Research Fellow, Institute of Forestry, Kneza Višeslava 3, 11000 Belgrade, Serbia, mara.tabakovic@gmail.com 
Entomophaga maimaiga imali su dominantnu ulogu u smanjenju brojnosti gubara $u$ stadijumu larve.

Ključne riječi: gubar, predatori, parazitoidi, patogeni

\section{INTRODUCTION}

The gypsy moth (Lymantria dispar L.), insect in the order Lepidoptera, is one of the most dangerous pests of broadleaf forests and orchards. It is characterised by a high reproductive capacity, considerable ecological plasticity and polyphagia. It occurs periodically in high numbers (outbreak). Although it is found on four continents (North Africa, Asia, Europe, North America), the greatest damage is caused to the forests of the Balkan Peninsula, which have all favourable environmental conditions for the gypsy moth development, and it often occurs in outbreaks.

Biological control, as the part of the forest integrated protection, is defined as the use of natural enemies (parasitoids, predators, and pathogens) to regulate or control pests. Various strategies have been used for the enhancement of biological control agents. Classical biological control is simply a special case of a general pattern in which populations are regulated by density-dependent processes, a major class of which involves predator-prey or parasitoid-host interactions.

Recent emphasis on the development of an integrated control program for the gypsy moth has necessitated an understanding of its mortality-causing biological agents. Throughout the holarctic region there is a wide range of natural enemies of this insect. The diseases caused by viruses, bacteria or fungi contribute to the decline of gypsy moth populations. For example, baculovirus - Lymantria dispar nucleopolyhedrosis virus ( $L d \mathrm{NPV})$ is specific to the gypsy moth, the most devastating natural disease, and it causes a dramatic collapse of outbreak populations by killing both the larvae and pupae. Infection by $L d \mathrm{NPV}$ is the most common source of mortality in high density populations and $L d \mathrm{NPV}$ epizootics, since it usually causes the collapse of host populations (Evans, 1986; McCoy et al., 1988).

Second example is the entomopathogenic fungus Entomophaga maimaiga Humber, Shimazu \& Soper (Entomophtorales: Entomophtoraceae) which was isolated and described as the natural enemy of the gypsy moth in Japan, where it causes the periodical epizootias. It is also spread in some parts of China and the Russian Far East (Hajek et al., 2005). In spite of the fact that it was introduced in North America in 1910-1911 (Speare and Colley, 1912), its presence in the natural populations of gypsy moth was determined only in 1989 (Hajek et al., 1996), when the pathogen caused pandemic in several countries (Andreadis and Weseloh, 1990; Hajek et al., 1990; Reardon and Hajek, 1998; Smitley et al., 1996). Today Entomophaga maimaiga is a very significant pathogen of gypsy moth in North America and Canada (Balser and Baumgard, 2001; Hajek, 1997; Hajek et al., 2005; Hoover, 2000; Howse and Scarr, 2002).

Bulgaria has been the third country in the world and the first one in Europe in which Entomophaga maimaiga was introduced successfully (Pilarska et al., 
2000). The first epizootics of it occurred in 2005 (Georgiev et al., 2007, 2010; Pilarska et al., 2006). This species is also present in oak forests in some Serbian regions (Tabaković-Tošić et al., 2012a, 2013- in press).

This paper presents the author's published and unpublished results of survey of the natural enemies (predators, parasitoids and pathogens) of the gypsy moth in Central Serbian forests, supplemented by available published results of other authors.

\section{MATERIALS AND METHODS}

Central Serbia, the area where the occurrence, population size and intensity of the adverse effects of the natural hosts of the gypsy moth were studied, is located in the centre and southeastern part of Republic of Serbia. It covers an area of about 55,000 square kilometers. It is located between 42 and 45 degrees northern latitude and between 19 and 23 degrees eastern longitude.

In Central Serbia, forests cover an area of 2.1 million hectares $(37,6 \%$ of the total area), of which $51 \%$ is the state property and $49 \%$ is the private property, timber volume amounts to 333 million $\mathrm{m}^{3}(59.2 \%$ in state forests and $40.8 \%$ in private forests), and volume increment is $3.9 \mathrm{~m}^{3} / \mathrm{ha}^{-1}$ (56.6\% in state and $43.4 \%$ in private forests); broadleaves account for $86.9 \%$ of the total timber volume (beech $43.9 \%$, oaks $26.3 \%$, other broadleaves 29.8\%) (Banković et al., 2009).

Every year over the last twenty years period, in all broadleaf forests, a survey of main predators, parasitoids and pathogens was conducted from April to late November during studies of the population dynamics and outbreaks of the gypsy moth (Tabaković-Tošić et al., 2002; Tabaković-Tošić, 2004, 2006, 2011).

The studies of the presence and density of the main predator species of the gypsy moth were conducted by using the methods, typical for some families to which the insects belong.

The detailed quantitative and qualitative studies of the parasitisation rate of the sampled egg masses were conducted in the laboratory of the Institute of Forestry, and, depending on the observed parameter, either ocular method or method of the survey by using binocular magnifier was applied. From each egg mass, 100 randomly sampled, previously cleaned eggs, were placed in the specially prepared test tubes. The emergence of the imago parasitoids was reported every day until the end of the process.

The field-collected larvae and pupae were grown under the laboratory conditions in the climate chamber. During the all laboratory experiments, temperature and light conditions were constant (temperature $21^{\circ} \mathrm{C}$, light regime - 8 hours night, 16 hours a day).

The studies of the presence of entomopathogenic viruses, bacteria and fungi in the dead gypsy moth larvae were conducted in the field and laboratory conditions. In the field conditions the characteristic symptoms of some diseases were identified by using ocular method, while in the laboratory conditions, they were identified by dissection of the dead larvae and the microscope survey (Tabaković-Tošić et al., 2012). 


\section{RESULTS AND DISCUSSION}

Natural enemy populations have the unique ability to interact with their prey or host populations and to regulate them at lower levels than would occur otherwise. Some are effective at extremely low prey levels, other only at higher levels.

In biological control parlance, natural enemies are referred to as parasitoids, predators or pathogens. Parasitoids may have one (univolutine), two or more generations to one of the host (multivolutine), and they tend to attack only one host stage, although there is also some overlapping in certain cases - adult insects do not serve as hosts very often (Debach, 1974). Predatory insects differ from parasitic ones since the larvae or nymphs, as the case may be, require several or many prey individuals to attain maturity.

Pathogenic microorganisms attack insects and have life cycles more or less characteristic of similar micro-organisms developing in other groups of animals. Insects are probably subject to as wide a variety of diseases. A number of pathogenic microorganisms - viruses, bacteria, fungi, and microsporidia (for example: Lymantria dispar nucleopolyhedrosis virus, Bacillus thuringiensis Berliner, Entomophaga maimaiga Humber, Shimazu \& Soper, Nosema lymantriae (Weiser), Nosema serbica Weiser) - infect the gypsy moth (Pilarska and Vávra, 1991; Sidor, 1979; Stiles et al., 1983; Tabaković-Tošić, 2008; Tabaković-Tošić et al., 2011a,b; Weiser, 1998). The epizootics of them are often spectacular, and mortality is most prevalent during gypsy moth outbreaks.

Based on the literature data, a total of 81 species which are natural enemies of the gypsy moth, i.e. 17 predators, 49 parasitoids, 10 saprophagous insects, and 5 pathogens, have been reported in Central Serbia (Table 1). The parasitoids which attack the larval instar of the gypsy moth are most frequent (31 species), followed by the predators of the gypsy moth eggs ( 12 species), the parasitoids of the pupae of the host (13 species), saprophages and parasitoids of the same instar (10 species), predators of the larvae 5 species), parasitoids of the gypsy moth eggs (5 species), pathogens of the larvae (5 species) (Table 1 ).

During the observed period, in the gypsy moth populations, the activity of 59 natural enemies of this insect - twenty-one predators, twenty-nine parasitoids, seven parasitoids or saprophagy and two pathogens -was reported. The gypsy moth eggs were attacked by thirteen species of the predators, larvae by six species, and larvae and pupae by two species.

There were three parasitoids species of the gypsy moth eggs, nineteen parasitoids species of the gypsy moth larvae, and seven parasitoids species of the gypsy moth pupae. Parasitoids or saprophages of gypsy moth pupae were represented by seven species. Two pathogenic species ( $L d \mathrm{NPV}$ and Entomophaga maimaiga) has been identified. (Table 1).

Table 1. Natural enemies of the gypsy moth in the forests of Central Serbia

\begin{tabular}{|l|l|l|l|}
\hline Gypsy moth & Significance \\
\hline Natural enemies & Instar & Literature sources & $\begin{array}{l}\text { Personal } \\
\text { research }\end{array}$ \\
\hline PREDATORS & egg & +++ & +++ \\
\hline Trombidium holosericeum (Linnaeus, 1758) & & \\
\hline
\end{tabular}




\begin{tabular}{|c|c|c|c|}
\hline \multicolumn{2}{|l|}{ Gypsy moth } & \multicolumn{2}{|l|}{ Significance } \\
\hline Natural enemies & Instar & Literature sources & $\begin{array}{l}\text { Personal } \\
\text { research }\end{array}$ \\
\hline [Acari: Trombiculidae] & & {$[9,11]^{*}$} & {$[6]^{* *}$} \\
\hline $\begin{array}{l}\text { Forficula auricularia Linnaeus, } 1758 \\
\text { [Dermaptera: Forficulidae] }\end{array}$ & egg & $\begin{array}{l}+++ \\
{[9,11]}\end{array}$ & $\begin{array}{l}+++ \\
{[6]}\end{array}$ \\
\hline $\begin{array}{l}\text { Dermestes lardarius Linnaeus, } 1758 \\
\text { [Coleoptera: Dermestidae] }\end{array}$ & egg & - & $\begin{array}{ll}++ \\
{[3]}\end{array}$ \\
\hline $\begin{array}{l}\text { Dermestes erichsoni Ganglbauer, } 1904 \\
\text { [Coleoptera: Dermestidae] }\end{array}$ & egg & $\begin{array}{l}+++ \\
{[11,12]}\end{array}$ & $\begin{array}{l}++ \\
{[1,2,3,6]}\end{array}$ \\
\hline $\begin{array}{l}\text { Megatoma pici Kalik, } 1952 \\
\text { [Coleoptera: Dermestidae] }\end{array}$ & egg & $\begin{array}{l}+++ \\
{[11,12]}\end{array}$ & $+1,2,3,6]$ \\
\hline $\begin{array}{l}\text { Megatoma pubescens (Zetterstedt, 1828) } \\
\text { [Coleoptera: Dermestidae] }\end{array}$ & egg & $\begin{array}{l}++ \\
{[11,12]}\end{array}$ & $\begin{array}{l}+ \\
{[6]}\end{array}$ \\
\hline $\begin{array}{l}\text { Megatoma undata (Linnaeus, 1758) } \\
\text { [Coleoptera: Dermestidae] }\end{array}$ & egg & $\begin{array}{l}++ \\
{[9,11]}\end{array}$ & $\begin{array}{l}+ \\
{[6]}\end{array}$ \\
\hline $\begin{array}{l}\text { Ctesias serra (Fabricius, 1792) } \\
\text { [Coleoptera: Dermestidae] }\end{array}$ & egg & {$[11,12]$} & - \\
\hline $\begin{array}{l}\text { Globicornis nigripes (Fabricius, 1792) } \\
\text { [Coleoptera: Dermestidae] }\end{array}$ & egg & {$[11,12]$} & $\begin{array}{l}+ \\
{[6]}\end{array}$ \\
\hline $\begin{array}{l}\text { Julistus floralis (Olivier, 1790) } \\
\text { [Coleoptera: Cantharidae] }\end{array}$ & egg & $\begin{array}{l}+++ \\
{[9,11,12]}\end{array}$ & + \\
\hline $\begin{array}{l}\text { Malachus bipustulatus (Linnaeus, 1758) } \\
\text { [Coleoptera: Cantharidae] }\end{array}$ & egg & $\begin{array}{l}+++ \\
{[11,12]}\end{array}$ & + \\
\hline $\begin{array}{l}\text { Podisus maculiventris (Say, 1832) } \\
\text { [Heteroptera: Pentatomidae] }\end{array}$ & egg & {$[11,12]$} & + \\
\hline $\begin{array}{l}\text { Formica rufa Linnaeus, } 1758 \\
\text { [Hymenoptera: Formicidae] }\end{array}$ & egg & $\begin{array}{l}++ \\
{[4,9]}\end{array}$ & $\begin{array}{l}+ \\
{[6]}\end{array}$ \\
\hline $\begin{array}{l}\text { Carabus latus Dejean, } 1826 \\
\text { [Coleoptera: Carabidae] }\end{array}$ & egg & - & {$[3,6]$} \\
\hline $\begin{array}{l}\text { Silpha quadripunctata Schreber, } 1759 \\
\text { [Coleoptera: Silphidae] }\end{array}$ & larvae & $\begin{array}{l}++ \\
{[11,12]}\end{array}$ & $\begin{array}{l}+++ \\
{[6]}\end{array}$ \\
\hline $\begin{array}{l}\text { Xylodrepa quadripunctata (Linnaeus, 1758) } \\
\text { [Coleoptera: Silphidae] }\end{array}$ & larvae & $\begin{array}{l}+ \\
{[9]}\end{array}$ & - \\
\hline $\begin{array}{l}\text { Carabus coriaceus (Linnaeus, 1758) } \\
\text { [Coleoptera: Carabidae] }\end{array}$ & larvae & - & $\begin{array}{l}+ \\
{[1,2,3,6]}\end{array}$ \\
\hline $\begin{array}{l}\text { Carabus cancellatus (Linnaeus, 1758) } \\
\text { [Coleoptera: Carabidae] }\end{array}$ & larvae & $\begin{array}{l}++ \\
{[9,12]}\end{array}$ & + \\
\hline $\begin{array}{l}\text { Carabus cavernosus Frivaldsky, } 1837 \\
\text { [Coleoptera: Carabidae] }\end{array}$ & larvae & - & $\begin{array}{l}+ \\
{[1,2,3,6]}\end{array}$ \\
\hline $\begin{array}{l}\text { Carabus intricatus (Linnaeus, 1758) } \\
\text { [Coleoptera: Carabidae] }\end{array}$ & larvae & - & $\begin{array}{l}++ \\
{[1,2,3,6]}\end{array}$ \\
\hline $\begin{array}{l}\text { Carabus scabriusculus bulgarus Lapouge, } \\
1908 \\
\text { [Coleoptera: Carabidae] }\end{array}$ & larvae & - & $\begin{array}{l}+ \\
{[1,2,3,6]}\end{array}$ \\
\hline $\begin{array}{l}\text { Calosoma sycophanta (Linnaeus, 1758) } \\
\text { [Coleoptera: Carabidae] }\end{array}$ & larvae/pupae & $\begin{array}{l}++ \\
{[5,9,12,19]}\end{array}$ & $\begin{array}{l}+++ \\
{[1,2,3,6]}\end{array}$ \\
\hline $\begin{array}{l}\text { Calosoma inquisitor (Linnaeus, 1758) } \\
\text { [Coleoptera: Carabidae] }\end{array}$ & larvae/pupae & {$[9,12]$} & $+1,2,6]$ \\
\hline \multicolumn{4}{|l|}{ PARASITOIDS } \\
\hline $\begin{array}{l}\text { Anastatus japonicus Ashmead, } 1904 \\
\text { [Hymenoptera: Eupelmidae] }\end{array}$ & egg & $\begin{array}{l}+++ \\
{[9,12,20,21]}\end{array}$ & $\begin{array}{l}++ \\
{[1,2,3,4,6]}\end{array}$ \\
\hline $\begin{array}{l}\text { Ooencyrtus kuwanae (Howard, 1910) } \\
\text { [Hymenoptera: Encyrtidae] }\end{array}$ & egg & $\begin{array}{l}+++ \\
{[9,12]}\end{array}$ & {$[1,2,3,4,6]$} \\
\hline $\begin{array}{l}\text { Ooencyrtus tardus (Ratzeburg, 1844) } \\
\text { [Hymenoptera: Encyrtidae] }\end{array}$ & egg & $\begin{array}{l}++ \\
{[9]}\end{array}$ & - \\
\hline $\begin{array}{l}\text { Ooencyrtus masii (Mercet, 1921) } \\
\text { [Hymenoptera: Encyrtidae] }\end{array}$ & egg & $\begin{array}{l}+ \\
{[9]}\end{array}$ & - \\
\hline $\begin{array}{l}\text { Eremioscelio lymantriae Masnil, } 1958 \\
\text { [Hymenoptera: Scelionidae] }\end{array}$ & egg & $\begin{array}{l}++ \\
{[9,12]}\end{array}$ & $\begin{array}{l}+ \\
{[6]}\end{array}$ \\
\hline $\begin{array}{l}\text { Casinaria tenuiventris (Gravenhorst, 1829) } \\
\text { [Hymenoptera: Ichneumonidae] }\end{array}$ & larvae & $\begin{array}{l}+ \\
{[9,12,20]}\end{array}$ & $\begin{array}{l}+ \\
{[6]}\end{array}$ \\
\hline
\end{tabular}




\begin{tabular}{|c|c|c|c|}
\hline \multicolumn{2}{|l|}{ Gypsy moth } & \multicolumn{2}{|l|}{ Significance } \\
\hline Natural enemies & Instar & Literature sources & $\begin{array}{l}\text { Personal } \\
\text { research }\end{array}$ \\
\hline $\begin{array}{l}\text { Phobocampe disparis (Viereck, 1911) } \\
\text { [Hymenoptera: Ichneumonidae] }\end{array}$ & larvae & $\begin{array}{l}++ \\
{[8,9,10,12,13,20]}\end{array}$ & $\begin{array}{l}+ \\
{[6]}\end{array}$ \\
\hline $\begin{array}{l}\text { Phobocampe pulchella (Thomson, 1887) } \\
\text { [Hymenoptera: Ichneumonidae] }\end{array}$ & larvae & $\begin{array}{l}+++ \\
{[9,12]}\end{array}$ & $\begin{array}{l}++ \\
{[6]}\end{array}$ \\
\hline $\begin{array}{l}\text { Apanteles glomeratus (Linnaeus, 1758) } \\
\text { [Hymenoptera: Braconidae] }\end{array}$ & larvae & $\begin{array}{l}+ \\
{[9]}\end{array}$ & {$[3,6]$} \\
\hline $\begin{array}{l}\text { Apanteles lacteicolor } \text { Viereck, } 1911 \\
\text { [Hymenoptera: Braconidae] }\end{array}$ & larvae & {$[9,10,12,23]$} & {$[3,6]$} \\
\hline $\begin{array}{l}\text { Cotesia melanoscela (Ratzeburg, 1844) } \\
\text { [Hymenoptera: Braconidae] }\end{array}$ & larvae & $\begin{array}{l}+++ \\
{[1,3,6,7,8,9,10,12,13,20,} \\
21,23]\end{array}$ & $\begin{array}{l}++ \\
{[1,2,3,6]}\end{array}$ \\
\hline $\begin{array}{l}\text { Cotesia ocneriae (Ivanov, 1898) } \\
\text { [Hymenoptera: Braconidae] }\end{array}$ & larvae & $+7,9,10,12,23]$ & $\begin{array}{l}++ \\
{[6]}\end{array}$ \\
\hline $\begin{array}{l}\text { Cotesia scabricula (Reinhard, 1880) } \\
\text { [Hymenoptera: Braconidae] }\end{array}$ & larvae & + & $\begin{array}{l}+ \\
{[6]}\end{array}$ \\
\hline $\begin{array}{l}\text { Cotesia spuria }(\text { Wesmael, 1837) } \\
\text { [Hymenoptera: Braconidae] }\end{array}$ & larvae & $+9,12]$ & - \\
\hline $\begin{array}{l}\text { Protapanteles liparidis (Bouček, 1834) } \\
\text { [Hymenoptera: Braconidae] }\end{array}$ & larvae & {$[1,8,9,12,20,23]$} & $\begin{array}{l}++ \\
{[1,2,3,6]}\end{array}$ \\
\hline $\begin{array}{l}\text { Protapanteles porthetrie Muesebeck, } 1954 \\
\text { [Hymenoptera: Braconidae] }\end{array}$ & larvae & $\begin{array}{l}+++ \\
{[6,9,10,12,17,20,21,23]}\end{array}$ & $\begin{array}{l}++ \\
{[6]}\end{array}$ \\
\hline $\begin{array}{l}\text { Protapanteles fulvipes (Haliday,1834) } \\
\text { [Hymenoptera: Braconidae] }\end{array}$ & larvae & {$[9,12]$} & $\begin{array}{l}+ \\
+ \\
{[6]}\end{array}$ \\
\hline $\begin{array}{l}\text { Meteorus versicolor }(\text { Wesmael, } 1835) \\
\text { [Hymenoptera: Braconidae] }\end{array}$ & larvae & {$[8,9,10,12]$} & $\begin{array}{l}+ \\
+ \\
{[6]}\end{array}$ \\
\hline $\begin{array}{l}\text { Meteorus gyrator (Thunberg, 1822) } \\
\text { [Hymenoptera: Braconidae] }\end{array}$ & larvae & $\begin{array}{l}+ \\
{[9]}\end{array}$ & - \\
\hline $\begin{array}{l}\text { Eulophus slovacus Bouček, } 1959 \\
\text { [Hymenoptera: Eulophidae] }\end{array}$ & larvae & $\begin{array}{l}+ \\
{[9]}\end{array}$ & - \\
\hline $\begin{array}{l}\text { Cirrospilus pictus (Nees, 1834) } \\
\text { [Hymenoptera: Eulophidae] }\end{array}$ & larvae & + & - \\
\hline $\begin{array}{l}\text { Elachertus charondas (Walker, 1839) } \\
\text { [Hymenoptera: Eulophidae] }\end{array}$ & larvae & $\begin{array}{l}+ \\
{[9]}\end{array}$ & - \\
\hline $\begin{array}{l}\text { Euplectrus liparidis Ferrière, } 1941 \\
\text { [Hymenoptera: Eulophidae] }\end{array}$ & larvae & $\begin{array}{l}+ \\
{[9]}\end{array}$ & $\begin{array}{l}++ \\
{[6]}\end{array}$ \\
\hline $\begin{array}{l}\text { Sympiesis sericeicornis (Nees, 1834) } \\
\text { [Hymenoptera: Eulophidae] }\end{array}$ & larvae & + & - \\
\hline $\begin{array}{l}\text { Exorista larvarum (Linnaeus, 1758) } \\
\text { [Diptera: Tachinidae] }\end{array}$ & larvae & {$[4,12,14,22]$} & $\begin{array}{l}+ \\
{[3,6]}\end{array}$ \\
\hline $\begin{array}{l}\text { Parasetigena silvestris (Robineau-Desvoidy, } \\
\text { 1863) } \\
\text { [Diptera: Tachinidae] }\end{array}$ & larvae & {$[4,12,13,14,15,17]$} & $\begin{array}{l}++ \\
+6]\end{array}$ \\
\hline $\begin{array}{l}\text { Phorocera agilis (Robineau-Desvoidy, 1830) } \\
\text { [Diptera: Tachinidae] }\end{array}$ & larvae & $\begin{array}{l}++ \\
{[9]}\end{array}$ & - \\
\hline $\begin{array}{l}\text { Blondelia nigripes (Fallen, } 1810 \text { ) } \\
\text { [Diptera: Tachinidae] }\end{array}$ & larvae & {$[1,9,12,16]$} & - \\
\hline $\begin{array}{l}\text { Compsilura concinnata (Meigen, 1824) } \\
\text { [Diptera: Tachinidae] }\end{array}$ & larvae & $\begin{array}{l}+++ \\
{[1,9,12,14,15,16,17,22]}\end{array}$ & $\begin{array}{ll}+ \\
{[3,6]}\end{array}$ \\
\hline $\begin{array}{l}\text { Drino inconspicua }(\text { Meigen, } 1830) \\
\text { [Diptera: Tachinidae] }\end{array}$ & larvae & {$[9,12,14,16,22]$} & - \\
\hline $\begin{array}{l}\text { Carcelia lucorum (Meigen, 1824) } \\
\text { [Diptera: Tachinidae] }\end{array}$ & larvae & + & - \\
\hline $\begin{array}{l}\text { Carcelia gnava (Meigen, 1824) } \\
\text { Syn. Senometopia separata (Rondani, 1859) } \\
\text { [Diptera: Tachinidae] }\end{array}$ & larvae & $+[9,12,16,22,24]$ & ++ \\
\hline $\begin{array}{l}\text { Senometopia susurrans (Rondani, 1859) } \\
\text { [Diptera: Tachinidae] }\end{array}$ & larvae & $\begin{array}{l}++ \\
{[9,12,14]}\end{array}$ & - \\
\hline $\begin{array}{l}\text { Zenillia libatrix (Panzer, 1798) } \\
\text { [Diptera: Tachinidae] }\end{array}$ & larvae & $\begin{array}{l}+ \\
{[9,16]}\end{array}$ & - \\
\hline
\end{tabular}




\begin{tabular}{|c|c|c|c|}
\hline \multicolumn{2}{|l|}{ Gypsy moth } & \multicolumn{2}{|l|}{ Significance } \\
\hline Natural enemies & Instar & Literature sources & $\begin{array}{l}\text { Personal } \\
\text { research }\end{array}$ \\
\hline $\begin{array}{l}\text { Blepharipa pratensis (Meigen, 1824) } \\
\text { [Diptera: Tachinidae] }\end{array}$ & larvae & $\begin{array}{l}+++ \\
{[1,4,9,12,14,16,17,22]}\end{array}$ & $\begin{array}{l}++ \\
{[1,2,3,6]}\end{array}$ \\
\hline $\begin{array}{l}\text { Blepharipa schineri (Mesnil, 1939) } \\
\text { [Diptera: Tachinidae] }\end{array}$ & larvae & $\begin{array}{l}++ \\
{[9,12,14,16,22]}\end{array}$ & $\begin{array}{l}+ \\
{[6]} \\
\end{array}$ \\
\hline $\begin{array}{l}\text { Gregopimpla inquisitor (Scopoli, 1763) } \\
\text { [Hymenoptera: Ichneumonidae] }\end{array}$ & pupae & $\begin{array}{l}+ \\
{[12,20]} \\
\end{array}$ & - \\
\hline $\begin{array}{l}\text { Acropimpla didyma (Gravenhorst, } 1829) \\
\text { [Hymenoptera: Ichneumonidae] }\end{array}$ & pupae & $\begin{array}{l}+ \\
{[9]}\end{array}$ & - \\
\hline $\begin{array}{l}\text { Apechtis compunctor (Linnaeus, 1758) } \\
\text { [Hymenoptera: Ichneumonidae] }\end{array}$ & pupae & $++[9,12,20]$ & - \\
\hline $\begin{array}{l}\text { Apechtis capulifera (Krichbaumer, 1887) } \\
\text { [Hymenoptera: Ichneumonidae] }\end{array}$ & pupae & $\left.\begin{array}{l}+ \\
+9\end{array}\right]$ & - \\
\hline $\begin{array}{l}\text { Apechtis rufata (Gmelin, 1790) } \\
\text { [Hymenoptera: Ichneumonidae] }\end{array}$ & pupae & + & - \\
\hline $\begin{array}{l}\text { Pimpla instigator Fabricius, } 1793 \\
\text { [Hymenoptera: Ichneumonidae] }\end{array}$ & pupae & $\begin{array}{l}++ \\
{[4,9,12,20,22]}\end{array}$ & $\begin{array}{l}+ \\
{[6]}\end{array}$ \\
\hline $\begin{array}{l}\text { Pimpla inquisitor (Scopoli, 1763) } \\
\text { [Hymenoptera: Ichneumonidae] }\end{array}$ & pupae & $\begin{array}{l}+ \\
{[9,12,20]}\end{array}$ & $\begin{array}{l}++ \\
{[6]}\end{array}$ \\
\hline $\begin{array}{l}\text { Pimpla turionellae (Linnaeus, 1758) } \\
\text { [Hymenoptera: Ichneumonidae] }\end{array}$ & pupae & {$[1,9,20,22]$} & $\begin{array}{l}++ \\
{[6]}\end{array}$ \\
\hline $\begin{array}{l}\text { Theronia atalantae (Poda, 1761) } \\
\text { [Hymenoptera: Ichneumonidae] }\end{array}$ & pupae & $\begin{array}{l}+++ \\
{[8,9,12,20,22]}\end{array}$ & + \\
\hline $\begin{array}{l}\text { Polytribax perspicillator (Gravenhorst, } 1807 \text { ) } \\
\text { [Hymenoptera: Ichneumonidae] }\end{array}$ & pupae & $\begin{array}{l}+ \\
{[9,12]}\end{array}$ & - \\
\hline $\begin{array}{l}\text { Lymantrichneumon disparis (Poda, 1761) } \\
\text { [Hymenoptera: Ichneumonidae] }\end{array}$ & pupae & $\begin{array}{l}+++ \\
{[4,12,20,22]}\end{array}$ & $\begin{array}{l}+ \\
{[1,2,3,6]}\end{array}$ \\
\hline $\begin{array}{l}\text { Brachimeria intermedia } \text { (Nees, 1834) } \\
\text { [Hymenoptera: Chalcididae] }\end{array}$ & pupae & $\begin{array}{l}+++ \\
{[12,20,22]}\end{array}$ & + \\
\hline $\begin{array}{l}\text { Brachimeria femorata (Panzer, 1798) } \\
\text { [Hymenoptera: Chalcididae] }\end{array}$ & pupae & $\begin{array}{l}++ \\
{[9,12]}\end{array}$ & + \\
\hline \multicolumn{4}{|l|}{ PARASITOIDS OR SAPROPHAGY? } \\
\hline $\begin{array}{l}\text { Agria affinis (Fallén, 1817) } \\
\text { [Diptera: Sarcophagidae] }\end{array}$ & pupae & $\begin{array}{l}+++ \\
{[9,12,14]}\end{array}$ & $\begin{array}{l}++ \\
{[6]}\end{array}$ \\
\hline $\begin{array}{l}\text { Agria monachae Kramer, } 1908 \\
\text { [Diptera: Sarcophagidae] }\end{array}$ & pupae & $\begin{array}{l}+ \\
{[9,12]}\end{array}$ & - \\
\hline $\begin{array}{l}\text { Kramerea schuetzei (Kramer, 1909) } \\
\text { [Diptera: Sarcophagidae] }\end{array}$ & pupae & $\begin{array}{l}++ \\
{[9,12,14,18]}\end{array}$ & $\begin{array}{l}+ \\
{[6]}\end{array}$ \\
\hline $\begin{array}{l}\text { Parasarcophaga harpax (Pandelle, 1896) } \\
\text { [Diptera: Sarcophagidae] }\end{array}$ & pupae & $\begin{array}{l}++ \\
{[9,12,14]}\end{array}$ & $\begin{array}{l}+ \\
{[6]}\end{array}$ \\
\hline $\begin{array}{l}\text { Parasarcophaga portshinskyi Rohdendorf, } \\
1937 \\
\text { [Diptera: Sarcophagidae] }\end{array}$ & pupae & $\begin{array}{l}++ \\
{[9,12,18]}\end{array}$ & $\begin{array}{l}+ \\
{[6]}\end{array}$ \\
\hline $\begin{array}{l}\text { Parasarcophaga tuberose (Pandelle, 1896) } \\
\text { [Diptera: Sarcophagidae] }\end{array}$ & pupae & $\begin{array}{l}+ \\
{[9,12,14,18]}\end{array}$ & - \\
\hline $\begin{array}{l}\text { Parasarcophaga uliginosa }(\text { Kramer, 1908) } \\
\text { [Diptera: Sarcophagidae] }\end{array}$ & pupae & + & $\begin{array}{l}+ \\
{[6]}\end{array}$ \\
\hline $\begin{array}{l}\text { Robineauella pseudoscoparia }(\text { Kramer, 1911) } \\
\text { [Diptera: Sarcophagidae] }\end{array}$ & pupae & + & - \\
\hline $\begin{array}{l}\text { Muscina pabulorum (Fallen, 1817) } \\
\text { [Diptera: Muscidae] }\end{array}$ & pupae & $\begin{array}{l}+ \\
{[9,12]}\end{array}$ & $\begin{array}{l}+ \\
{[6]}\end{array}$ \\
\hline $\begin{array}{l}\text { Muscina stabulans (Fallen, 1817) } \\
\text { [Diptera: Muscidae] }\end{array}$ & pupae & $\begin{array}{l}+ \\
{[9,12]}\end{array}$ & $\begin{array}{l}+ \\
+6] \\
\end{array}$ \\
\hline \multicolumn{4}{|l|}{ PATHOGENS } \\
\hline $\begin{array}{l}\text { Lymantria dispar nucleopolyhedrosis virus } \\
\text { [baculovirus] }\end{array}$ & larvae & $\begin{array}{l}+++ \\
{[9]}\end{array}$ & $\begin{array}{l}+++ \\
{[3,5,6]}\end{array}$ \\
\hline $\begin{array}{l}\text { Thelohania spp. } \\
\text { [Microsporidia: Thelohaniidae] }\end{array}$ & larvae/pupae & $\begin{array}{l}? \\
{[9]}\end{array}$ & - \\
\hline Vairimorpha $s p$. & larvae & $\begin{array}{l}? \\
{[2]}\end{array}$ & - \\
\hline
\end{tabular}




\begin{tabular}{|l|l|l|l|}
\hline Gypsy moth & Significance \\
\hline Natural enemies & larvae & $\begin{array}{l}? \\
{[2]}\end{array}$ & $\begin{array}{l}\text { Personal } \\
\text { research }\end{array}$ \\
\hline Endoreticulatus sp. & larvae & $\begin{array}{l}? \\
{[9]}\end{array}$ & - \\
\hline $\begin{array}{l}\text { Streptococcus spp. } \\
\text { [Lactobacillales: Streptococcaceae] }\end{array}$ & larvae & - & - \\
\hline $\begin{array}{l}\text { Entomophaga maimaiga } \text { Humber, Shimazu\& } \\
\text { Soper } \\
\text { [Entomophtorales: Entomophtoraceae] }\end{array}$ & - & + \\
\hline
\end{tabular}

Legend:

+ poorly represented species $\quad++$ represented species

+++ highly represented species

* [1] Đorović, [2] Glavendekić et al., 2006; [3] Maksimović, 1953; [4] Maksimović, 1973; [5] Maksimović \& Sivčev, 1980; [6] Maksimović \& Sivčev, 1984; [7] Maksimović \& Sivčev, 1987; [8] Marović \& Minić, 1987; [9] Mihajlović, 2008; [10] Minić, 1988; [11] Nonveiller, 1980; [12] Ristić et al.,1998; [13] Sisojević, 1953; [14] Sisojević, 1955; [15] Sisojević, 1959; [16] Sisojević, 1975; [17] Sisojević \& Vasić, 1980; [18] Sisojević et al., 1989; [19] Tomić \& Janković, 1973; [20] Vasić, 1958; [21] Vasić \& Salatić, 1959; [22] Vasić \& Sisojević, 1958; [23] Vasić \& Minić, 1980; [24] Drea, J.J., 1981)

** Unpublished and published data: [1] Tabaković-Tošić, 2006; [2] Tabaković-Tošić and Jovanović, 2007; [3] Tabaković-Tošić, 2011; [4] Tabaković-Tošić et al., 2011a; [5] Tabaković-Tošić et al, 2012; [6] Tabakovic-Tosic et al., in press)

Regarding the density of some predator species, Trombidium holosericeum, Forficula auricularia, Silpha quadripunctata, Calosoma sycophanta and Carabus $s p$. were most abundant ones. Calosoma sycophanta, which regularly occurs during the outbreak of the gypsy moth, was found more frequently than other predator species, and it reduced the population size of the gypsy moth both in the larval and imago instars.

Table 2. The laboratory analysis of the gypsy moth egg masses and Quantitative ratio of two species of parasitoids

\begin{tabular}{|c|c|c|c|c|c|c|c|c|}
\hline \multirow{3}{*}{ Year } & \multicolumn{2}{|c|}{ Number of } & \multicolumn{4}{|c|}{$\begin{array}{c}\text { Average number of fertilized eggs } \\
\text { in egg mass }\end{array}$} & \multicolumn{2}{|c|}{ Parasitoids } \\
\hline & \multirow[b]{2}{*}{ localities } & \multirow[b]{2}{*}{ egg mass } & \multicolumn{2}{|c|}{ Vital } & \multicolumn{2}{|c|}{ Parasited } & \multirow[t]{2}{*}{ Ooencyrtus kuwanae (\%) } & \multirow{2}{*}{$\begin{array}{c}\text { Anastatus } \\
\text { japonicus (\%) }\end{array}$} \\
\hline & & & $\mathbf{N}$ & $\%$ & $\mathbf{N}$ & $\%$ & & \\
\hline 1993 & 27 & 270 & 298.9 & 86.4 & 44.6 & 12.9 & $100^{*}$ & 0 \\
\hline 1994 & 35 & 350 & 555.9 & 95.0 & 20.5 & 3.5 & $100^{*}$ & 0 \\
\hline 1995 & 69 & 690 & $\begin{array}{l}543.8 \\
\end{array}$ & 98.7 & 2.8 & 0.5 & $100^{*}$ & 0 \\
\hline 1996 & 427 & 4270 & 348.4 & 85.9 & 43.8 & 10.8 & - & - \\
\hline 1997 & 725 & 7250 & 277.7 & 80.7 & 41.6 & 12.1 & - & - \\
\hline 2002 & 70 & 700 & 477.6 & 95.5 & 17.3 & 3.5 & 78 & 22 \\
\hline 2003 & 431 & 4310 & 570.7 & 88.3 & 79.9 & 11.2 & 69 & 31 \\
\hline 2004 & 1023 & 10230 & 386.1 & 79.2 & 97.4 & 20.0 & 73 & 27 \\
\hline 2005 & 266 & 2660 & 162.5 & 82.4 & 32.0 & 16.2 & 71 & 29 \\
\hline 2009 & 58 & 580 & 502.5 & 91.6 & 42.3 & 7.7 & 91 & 9 \\
\hline 2010 & 80 & 800 & 526.9 & 90.9 & 48.3 & 8.3 & 87 & 13 \\
\hline 2011 & 120 & 1200 & 465.8 & 92.2 & 34.5 & 6.9 & 90 & 10 \\
\hline
\end{tabular}

* from annual reports of Serbian report-diagnose-forecast service in the domain of forest protection

The dynamics of the emergence of the imago parasitoids were studied in the special laboratory experiment. Regarding the species of egg parasitoids, in the investigated period Anastatus japonicus accounted from 0 to 31\%, Ooencyrtus kuwanae $69-100 \%$ (Table 2). The average parasitism rate should not be taken as the final one, because under these laboratory conditions it is impossible to study 
all the effects of a range of parasitoids and predators to which the the egg masses are exposed in the field.

At the selected sites the cocoons of the parasitoids species from the families Braconidae and Tachinidae (Table 1) were regularly found in spring. Some species from Braconidae family (Apanteles genus) were most active in the parts of the forests with the highest insolation and whence the outbreak of the gypsy moth usually begins. Other species were considerably less frequent and were found individually (Tabakovic-Tosic et al., in press).

The activity of $L d \mathrm{NPV}$ was reported at many sites, characterized by the extremely high population size of the gypsy moth (Tabaković-Tošić et al., 2012, Tabakovic-Tosic et al., in press).

The higher mortality rate of the older gypsy moth larval instars in 2011 and 2012 was reported in the forest complexes of Belgrade and Valjevo (Tabaković-Tošić et al., 2012, Tabakovic-Tosic et al., in press), Donji Milanovac and Negotin (new localities - unpublished results) region, in the culmination phase of the new outbreak of the gypsy moth in Serbia. By field and laboratory studies of the causes of their death, the presence of azygospores and conidiospores of the entomopathogenic fungus Entomophaga maimaiga was reported in the dead caterpillars. It showed to be a powerful reducer of the population size of the gypsy moth, and in both regions it caused the collapse of the outbreak in 2011 and 2012.

\section{CONCLUSIONS}

A total of 88 species which are natural enemies of the gypsy moth, i.e. 23 predators, 49 parasitoids insects, 10 saprophagous insects, and 6 pathogens, has been reported in Central Serbia. The insects which attack the larval instar of the gypsy moth are most frequent followed by the predators of the gypsy moth eggs and the parasitoids of the pupae of the host. Regarding the number of the species, the representatives of the Hymenoptera (14 species from Ichneumonidae family and 11 species from Braconidae family) and Diptera orders (12 species from Tachinidae family and 8 species from Sarcophagidae family) are most frequent. Regarding the predators of the gypsy moth, Carabidae family, from Coleoptera order is most frequent.

In spite of such a great number of the natural hosts, outbreaks of the gypsy moth are still the regular and frequent phenomenon in the forests of central Serbia, which points to the fact that nature is not so powerful regarding this economically most harmful species of the broadleaf forests in central Serbia. For the time being, the only exception to this rule is entomopathogenic fungus Entomophaga maimaiga.

\section{Acknowledgments}

The study was partly financed by the Ministry of Science of the Republic of Serbia, the Project 31070 - SUBPROJECT: New technological methods in the integral protection of forests with the focus on the entomopathogenic fungus Entomophaga maimaiga, as the possible solution to the problem regarding the 
frequent occurrences of the outbreak of gypsy moth in the forest ecosystems of Serbia.

The author is particularly grateful to the Professor Dr Miloje Brajkovic [\%], from the University of Belgrade - Faculty of Biology, and to the Professor Dr Georgi Georgiev, from the Bulgarian Academy of Sciences - Forest Research Institute, for their great help during the determination of the above parasitic and pathogen species.

\section{LITERATURE}

Andreadis, T. and Weseloh, G. (1990): Discovery of Entomophaga maimaiga in North American gypsy moth, Lymantria dispar. Proc. Nat. Acad. Sci. US, 87: 2461-2465.

Balser, D., and Baumgard, A. (2001): Entomophaga: A New Tool in Gypsy Moth Management. http://www.hcs.ohio-state.edu/ODNR/Health/ONLAArticle97.html.

Banković, S., Medarević, M., Pantić, D. and Petrović, N. (2009): National Forest Inventory of the Republic of Serbia - Forests of the Republic of Serbia. Ministry of Agriculture, Forestry and Water Management - Forest Directory, 1-244, Belgrade. [in Serbian]

Debach, P. (1974): Biological control by natural enemies. Cambridge University Press, pp. 323, Cambridge.

Drea, J.J. (1981): Agricultural Research Service Sponsored Public Law 480 Project in Yugoslavia: 1972-75. - In: Doane C., M. McManus (Eds.): The Gypsy Moth: Research Toward Integrated Pest Management, U. S. Department of Agriculture, Washington D. C., 315-317.

Đorović, Đ. (1992): Biocenotic complex of oak defoliator larvae. Scientific book Monograph, book 1, Priština, 160-161. [in Serbian]

Evans, H.F. (1986): Ecology and epizootiology of Baculoviruses. In: Granados R. R. and Federici, B. A. (Ed-s), The Biology of Baculoviruses. Vol. II - Practical Application for Insect Control, CRC Press, 89-132, Boca Raton, Florida.

Georgiev, G., Mirchev, P., Pilarska, D., Golemanski, V., Pilarski, P., Tomovski, H. and Bochev, N. (2007): The gypsy moth will be neutralized. Forestry, 5: 8-10, Sofia. [in Bulgarian]

Georgiev, G., Pilarska, D., Mirchev, P., Rossnev, B., Petkov, P., Pilarski, P., Golemanski, V., Todorov, M., Takov, D., Hubenov, Z., Georgieva, M., Matova M. and Kitanova, S. (2010): Entomophaga maimaiga - a factor for increasing stability and enhancing biodiversity in oak forests on the Balkan Peninsula. In: Institute of Forestry, Belgrade (Eds) Proceedings of International Scientific Conference - Forest ecosystems and climate changes, Institute of Forestry, Belgrade, 1: 181-185, Belgrade.

Glavendekić, M., Pilarska, D., Mihajlović, Lj., Linde, A., Kolling, T. and Hoch, G. (2007): Microsporidian infections in Lymantria dispar L. (Lepidoptera, Lymantriidae) populations in Serbia. In: Institute of Forestry, Belgrade (Ed-s) Proceedings of International Scientific Conference - Sixty years of Institute of Forestry, Belgrade, Serbia - Sustainable use of forest ecosystems, (Rakonjac, Lj. Ed.), 8-10 October 2006, Donji Milanovac, Serbia, 275279. 
Hajek, A.E. (1997): Ecology of terrestrial fungal enthomopathogens. Adv. Microb. Ecol., 15: 193-249.

Hajek, A.E., Elkinton, J.S. and Witcosky, J.J. (1996): Introduction and spread of the fungal pathogen Entomophaga maimaiga (Zygomycetes: Entomophthorales) along the leading edge of gypsy moth (Lepidoptera: Lymantriidae) spread. Environ. Entomol., 25: 12351247.

Hajek, A.E., Humber, R.A., Elkinton, J., Walsh, S. and Silver, J. (1990): Allozyme and restriction fragment length polymorphism analyses confirm Entomophaga maimaiga responsible for 1989 epizootics in North American gypsy moth populations. - In: Proc. Natl. Acad. Sci. USA, 87: 6979-6982.

Hajek, A.E., McManus, M. and Delalibera, J.I. (2005): Catalogue of Introductions of Pathogens and Nematodes for Classical Biological Control of Insects and Mites. FHTET, USDA Forest Service, pp. 59, Morgantown.

Hoover, G.A. (2000): Gypsy Moth. Penn State College of Agricultural Sciences, Entomological Notes. http://www.ento.psu.edu/extension/factsheets/gypsymoth.htm.

Howse, G.M. and Scarr, T. (2002): Status of important forest insects in Ontario in 2002. Report prepared for the annual Forest Pest Management Forum, November 13-15, pp. 24, Ottawa.

Maksimović, M. (1953): Some observations about gradation crisis of gypsy moth in lowland and mountain forests in 1950. Plant Protection, Belgrade, 15:12-27. [in Serbian]

Maksimović, M. (1973): Follow-up of the parasite-predator complex in a zone of microbiological control of gypsy moth. Plant Protection, Belgrade, Vol. XXIV, 124125:265-268.

Maksimović, M. and Sivčev, I. (1980): A contribution to the investigation of the numerical increase of population of natural enemies of gypsy moth (Lymantria dispar L.) in forests. Plant Protection, Belgrade, 153:229-238. [in Serbian]

Maksimović, M. and Sivčev, I. (1984): Further studies on the numerical increase of natural enemies of the gypsy moth (Lymantria dispar L.) in forests. Zeitschrift für Angewandte Entomologie, Hamburg, H 4, 98:332-343.

Maksimović, M. and Sivčev, I. (1987): A contribution to the biological control of the gypsy moth (Lymantria dispar L.). Plant Protection, Belgrade, 181:197-205. [in Serbian]

Marović, R. and Minić, D. (1987): Contribution to the study of the state of gypsy moth on permanent test plots in Šmadija. Institute of Forestry and Woodworking Industry, Belgrade, Collection, 28-29:219-230. [in Serbian]

McCoy, C.W., Samson, R.A. and Boucias, D.G. (1988): Entomogenous fungi. In: Handbook of Natural Pesticides, Vol. V - Microbial Insecticides, Part A: Entomogenous Protozoa and Fungi (Ignoffo CM and Mandava NB, Eds.), CRC Press, 151-236, Boca Raton, Florida.

Mihajlović, Lj., (2008): The Gypsy Moth (Lymantria dispar L.) (Lepidoptera, Lymantridae) in Serbia. Sumarstvo (Forestry), 1-2: 1-26, Belgrade. [in Serbian]

Minić, D. (1988): Parasites of gypsy moth from Apanteles sp. (Hymenoptera, Braconidae) with special regard to Apanteles solitarius Ratzeburg and a possibility of its application in biological control of the host. PhD Thesis defended at the Faculty of forestry, Belgrade, 370 p. [in Serbian] 
Nonveiller, G. (1959): Predators of gypsy moth egg masses recorded in Yugoslavia during gypsy moth outbreak in the period 1945-1950. Plant Protection, Belgrade, 52-53:15-35. [in Serbian]

Pilarska, D., McManus, M., Hajek, A., F. Herard, Vega, F., Pilarski, P. and Markova, G. (2000): Introduction of the entomopathogenic fungus Entomophaga maimaiga Hum., Shim. \& Sop. (Zygomycetes:Entomophtorales) to a Lymantria dispar (L.) (Lepidoptera: Lymantriidae) population in Bulgaria. Pest Science, 73: 125-126.

Pilarska, D., McManus, M., Pilarski, P., Georgiev, G., Mirchev, P. and Linde, A. (2006): Monitoring the establishment and prevalence of the fungal entomopathogen Entomophaga maimaiga in two Lymantria dispar L. populations in Bulgaria. Pest Science, 79: 63-67.

Pilarska, D. and Vávra, J. (1991): Morphology and development of Nosema serbica Weiser, 1963 (Microspora, Nosematidae), parasite of the gypsy moth Lymantria dispar (Lepidoptera, Lymantriidae). Folia Parasitol., 38: 115-121.

Reardon, R. and Hajek, A. (1998): The Gypsy Moth Fungus Entomophaga maimaiga in North America. USDA Forest Service FHTET-97-11 June 1998.

Ristić, M., Sisojević, P. and Brajković, M. (1998): Parasitoids, Hyperparasitoids and Predators of Gypsy Moth (Lepidoptera, Lymantridae) in Yugoslav Countries. In: The Gypsy Moth Outbreaks in Serbia, Acta Entomologyca Serbica, Special issue, 13-37, Belgrade.

Sidor, C. (1979): The role of insect pathogenic microorganisms in the protection of the environment. Mikrobiologija (Microbiology), 16: 173-186, Belgrade.

Sisojević, P. (1953): Contribution to the knowledge of the role of tachinids as regulators of gypsy moth population in 1950. Journal of Serbian Academy of Science and Arts, 4: 63-92. [in Serbian]

Sisojević, P. (1955): Contribution to the knowledge of tachinid parasites of the gypsy moth in Yugoslavia. Plant Protection, Belgrade, 28: 3-10. [in Serbian]

Sisojević, P. (1959): Ecological studies of tachinid parasites of the gypsy moth (Annual report, 1958). Plant Protection, Belgrade, 52-53: 165-166. [in Serbian]

Sisojević, P. (1975): Population dynamics of tachinid parasites of the gypsy moth ( $L$. dispar L.) during a gradation period. Plant Protection, Belgrade, 132: 97-170. [in Serbian]

Sisojević, P., Čepelak, J. and Slamečkova, M. (1989): Contribution of the fauna of Sarcophagidae (Diptera) of Yugoslavia. Biosystematics, Belgrade, Vol. 15, 1:71-79. [in Serbian]

Sisojević, P. and Vasić, K. (1980): Parasites and hyperparasites of the gypsy moth and their role in host population reduction in Yugoslavia. Second annual scientific conference of the Entomological Society of Serbia. Abstract, 10-11, Belgrade. [in Serbian]

Smitley, D.R., Bauer, L.S., Hajek, A.E., Sapio, F.J. and Humber, R.A. (1996): Introduction and establishment of Entomophaga maimaiga, a fungal pathogen of Gypsy moth (Lepidoptera: Lymantriidae) in Michigan. Environ. Entomol., 24(6): 1685-1695.

Speare, A.T. and Colley, R.H. (1912): The artificial use of the brown-tail fungus in Massachusetts with practical suggestions for private experiments, and a brief note on a fungous disease of the gypsy caterpillar. Wright \& Potter, Boston. 
Stiles, B., Burand, P.J., Meda, M. and Wood, A.H. (1983). Characterization of Gypsy Moth (Lymantria dispar) Nuclear Polyhedrosis Virus. Applied and Environmental Microbiology, 46(2): 297-303.

Tabaković-Tošić, M. (2004): Gypsy moth population control. In: gypsy moth (Lymantria dispar L.) the most dangerous pests of our forests and orchards. Ministry of Agriculture, Forestry and Water Management, 25-26, Belgrade. [in Serbian]

Tabaković-Tošić, M. (2006): Monitoring abundance, population vitality and control of gypsy moth (Lymantria dispar L.) in Serbia during 2003-2005. Plant Doctor, XXXIV (3): 209-217, Novi Sad, Serbia.

Tabaković-Tošić, M. (2008): Entomopathogenic bacterium Bacillus thuringiensis ssp. kurstaki the important component of the integral protection of forest ecosystems. Institute of forestry Belgrade, special editions, 1-148, Belgrade. [in Serbian/English]

Tabaković-Tošić, M. (2011): Gypsy moth predators, parasites and pathogens in Belgrade forests in the period 2010-2011. Collection 63-64:101-111, Belgrade.

Tabaković-Tošić, M., Tošić, D., Marković, M., Mladenović, K., Radulović, Z. and Rajković, S. (2011a): Gypsy moth outbreaks in forest complexes of the Belgrade region in the period 1996-2011. Sustainable Forestry, Collection 63-64:113-122, Belgrade.

Tabaković-Tošić, M., Golubović Ćurguz, V. and Tošić, D. (2011b): New technological methods in the integrated forest protection in the Republic of Serbia. In: Institute of Plant Protection, Minsk (Ed-s) Proceedings of International scientific conference Integrated plant protection - Strategy and tactics, Institute of Plant Protection, Minsk, 49-55, Minsk.

Tabaković-Tošić, M., Georgiev, G., Mirchev, P., Tošić, D. and Golubović-Ćurguz, V. (2012): Entomophaga maimaiga - new entomopathogenic fungus in the Republic of Serbia. African Journal of Biotechnology, 11(34): 8571-8577.

Tabaković-Tošić, M., Georgiev, G., Mirchev, P., Tošić, D., Golubović-Ćurguz, V. (in press): Gypsy Moth in Central Serbia over the Previous Fifty Years. Acta Zoologica Bulgarica.

Tabaković-Tošić, M., Lazarev, V., Jančić, G. (2002): Economically harmful insects and phytopathogenic fungi in forests of Serbia 2001" SE "Srbijašume" Institute of Forestry, special edition, 1-127, Belgrade, Serbia.

Tabaković-Tošić, M. \& Jovanović, V. (2007): Gypsy moth (Lymantria dispar L.) outbreaks in the Republic of Serbia 2003-2005. Plant protection (FYUR Macedonia), 17: 213-224, Skopje.

Tomić, D. \& Janković, Lj. (1973): Comparative study of population levels of gypsy moth (Lymantria dispar L.) and its most important predator (Calosoma sycophanta L.) in Serbia and Macedonia, 1967-1972. Proceedings of the First Congress of Yugoslav Ecologists, Belgrade, 123-124. [in Serbian]

Vasić, K. (1958): Parasitic Hymenoptera of gypsy moth. Plant Protection, Belgrade, 4142:17-21. [in Serbian]

Vasić, K. \& Minić, D. (1976): Fauna of hyperparasites in cocons of solitary Apanteles species and Meteorus versicolor Nees., of primary parasites of the gypsy moth (Porthetria dispar L.) and the satin moth (Leucoma salicis L.) in Yugoslavia. Arch. Biol. Sciences, Belgrade, 28(3-4): 175-188. [in Serbian] 
Vasić, K. \& Salatić, S. (1959): A new contribution to the knowledge of the parasitic Hymenoptera of gypsy moth. Plant Protection, Belgrade, 52:47-50. [in Serbian]

Vasić, K. \& Sisojević, P. (1958): Parasites of pronymphs and pupae of the gypsy moth in Yugoslavia in 1957. Plant Protection, Belgrade, 41-42:49-52. [in Serbian]

Weiser, J. (1998): Pathogens of the Gypsy Moth in Central Europe: Host Range and Interactions. In: Proceedings - Population Dynamics, Impacts, and Integrated Management of Forest Defoliating Insects, M.L. McManus and A.M. Liebhold (eds), 322-333.

\title{
GYPSY MOTH, Lymantria dispar (L.), AND ITS NATURAL ENEMIES IN THE FORESTS OF CENTRAL SERBIA
}

\author{
Mara TABAKOVIĆ-TOŠIĆ
}

\section{Summary}

Based on the literature data, a total of 81 species which are natural enemies of the gypsy moth, i.e. 17 predators, 49 parasitoids insects, 10 saprophagous insects, and 5 pathogens, have been reported in Central Serbia. The parasitoids which attack the larval instar of the gypsy moth are most frequent, followed by the predators of the gypsy moth eggs and the parasitoids of the pupae of the host. Regarding the number of the species, the representatives of the Hymenoptera (14 species from Ichneumonidae family and 11 species from Braconidae family) and Diptera orders (13 species from Tachinidae family and 8 species from Sarcophagidae family) are most frequent. Regarding the predators of the gypsy moth, Carabidae family, from Coleoptera order, is most frequent.

During the twenty-year observed period, in the gypsy moth populations, the activity of 59 natural enemies of this insect - twenty-one predators, twenty-nine parasitoids, seven parasitoids or saprophages and two pathogens was reported. Regarding the density of some species, the most abundant predators were Trombidium holosericeum, Forficula auricularia, Silpha quadripunctata, Calosoma sycophanta and some species from genus Carabus, while the most abundant parasitoids were Ooencyrtus kuwanae (Howard) and Anastatus japonicus Ashmead, and pathogens Lymantria dispar nucleopolyhedrosis virus and Entomophaga maimaiga .

In the observed period, average parasitism rate of eggs in egg masses ranged from 0,5 to $20 \%$. The greatest positive change in the activity of the parasitoids occurred during the second outbreak, from $3,5 \%$ in 2002 to $20 \%$ in 2004 . Regardiong the species of egg parasites, in laboratory conditions Oencyrtus kuwanae was absolutely dominant (69100\%). In addition, at some sites Lymantria dispar nucleopolyhedrosis virus and Entomophaga maimaiga had the dominant role in the reduction of the gypsy moth density.

The activity of Lymantrya dispar NPV was reported at many sites, characterized by the extremely high population size of the gypsy moth. During the growing season in 2011 and 2012, in some forest areas of central Serbia, where the increased mortality rate of the gypsy moth larvae was reported and where there was no significant damage of the foliage caused by the feeding of them, the intensive research of the possible causes of this condition was done.

By field and laboratory studies of the causes of their death, the presence of azygospores and conidiospores of the entomopathogenic fungus Entomophaga maimaiga was reported in the dead caterpillars. It have been the first records of this kind is Serbia, i.e. Serbia is the third European country in which this fungus has been reported. In the investigated area, it showed to be a powerful reducer of the population size of the gypsy moth. 


\title{
GUBAR, Lymantria dispar (L.), I NJEGOVI PRIRODNI NEPRIJATELJI U ŠUMAMA CENTRALNE SRBIJE
}

\author{
Mara TABAKOVIĆ-TOŠIĆ
}

\section{Rezime}

Prema literaturnim podacima, u centralnoj Srbiji do danas je utvrđeno ukupno 81 vrsta prirodnih neprijatelja gubara, i to 17 predatora, 49 parazitoia, 10 vrsta koje se ponašaju kao saprofagi i parazitoidi, te 5 patogena. U navedenom broju najzastupljeniji su insekti koji parazitiraju larveni stadijum gubara, a na drugom mestu su predatori jaja i parazitoidi lutki domaćina. Po broju zastupljenih vrsta, najviše je pripadnika redova Hymenoptera (14 vrsta iz familije Ichneumonidae i 11 iz familije Braconidae) i Diptera (13 vrsta iz familije Tachinidae i 8 iz Familije Sarcophagidae). Od predatora gubara, najzastupljenija ja familija Carabidae iz reda Coleoptera.

Tokom dvadesetogodišnjeg istraživačkog perioda u populacijama gubara, uočena je pojačana aktivnost 59 vrsta njegovih prirodnih neprijatelja $\mathrm{i}$ to dvadeset jednog predatora, dvadest devet parazitoida, 7 parazitoida ili saprofaga i 2 patogena. Od svih nađenih vrsta, najveću brojnost i najjaču aktivnost su imali predatori Trombidium holosericeum, Forficula auricularia, Silpha quadripunctata, Calosoma sycophanta i pojedine vrste roda Carabus, parazitoidi Oencyrtus kuwanae i Anastatus japonicus, te patogeni $L d \mathrm{NPV}$ i Entomophaga maimaiga.

U posmatranom periodu, prosečna stopa parazitiranosti jaja u jajnim leglima kretala se od 0,5 do $20 \%$. Najveća pozitivna promena u aktivnosti parazitoida dogodila se tokom druge gradacije, od $3,5 \%$ u 2002. do $20 \%$ u 2004. godini. Kada je reč o vrstama janih parazitoida dobijenih u laboratorijskim uslovima gajenja jaja gubara, apsolutno dominantan bio je Oencyrtus kuwanae (69-100\%).

Aktivnost Lymantrya dispar NPV je registrovana na većem broju lokaliteta gde je brojnost gubara beležila enormne vrednosti. Tokom vegetacione sezone 2011. I 2012. godine, u pojedinim šumskim područjima centralne Srbije, gde je uočena veća smrtnost larvi gubara i gde nije došlo do značajnijeg oštećivanja lisne mase prouzrokovanog njihovom ishranom, obavljena su intenzivna istraživanja mogućih uzroka ovakvog stanja. Terenskim i laboratorijskim ispitivanjima uzroka smrtnosti starijih larvenih stupnjeva gubara, dokazano je prisustvo azigospora i konidiospora entomopatogene gljive Entomophaga maimaiga u uginulim gusenicama. Ovo su bili prvi nalazi ove vrste u Srbiji, odnosno, Srbija je treća zemlja u Evropi u kojoj je ova gljiva registrovana. Na istraživanim lokalitetima se pokazala kao moćan reduktor nivoa populacije gubara. 
